\title{
The use of diagnostic imaging for identifying abnormal gas accumulations in cetaceans and pinnipeds
}

\author{
Sophie Dennison ${ }^{1}$, Andreas Fahlman ${ }^{2}$ and Michael Moore ${ }^{3}$ \\ ${ }^{1}$ Marine Mammal Radiology, San Francisco, CA, USA \\ 2 Department of Life Sciences, Texas A\&M University - Corpus Christi, Corpus Christi, TX, USA \\ ${ }^{3}$ Marine Research Facility, Woods Hole Oceanographic Institution, Woods Hole, MA, USA
}

\section{Edited by:}

Michael Castellini, University of

Alaska Fairbanks, USA

\section{Reviewed by:}

Paul Ponganis, Scripps Instituion of

Oceanography, USA

Jo-Ann Mellish, Alaska SeaLlfe

Center, USA

Todd O'Hara, University of Alaska

Fairbanks, USA

*Correspondence:

Sophie Dennison, Marine Mammal Radiology, 851 Indiana Street \#307, San Francisco, CA 94107, USA.

e-mail: vetradiologist@hotmail.com
Recent dogma suggested that marine mammals are not at risk of decompression sickness due to a number of evolutionary adaptations. Several proposed adaptations exist. Lung compression and alveolar collapse that terminate gas-exchange before a depth is reached where supersaturation is significant and bradycardia with peripheral vasoconstriction affecting the distribution, and dynamics of blood and tissue nitrogen levels. Published accounts of gas and fat emboli and dysbaric osteonecrosis in marine mammals and theoretical modeling have challenged this view-point, suggesting that decompression-like symptoms may occur under certain circumstances, contrary to common belief. Diagnostic imaging modalities are invaluable tools for the non-invasive examination of animals for evidence of gas and have been used to demonstrate the presence of incidental decompression-related renal gas accumulations in some stranded cetaceans. Diagnostic imaging has also contributed to the recognition of clinically significant gas accumulations in live and dead cetaceans and pinnipeds. Understanding the appropriate application and limitations of the available imaging modalities is important for accurate interpretation of results. The presence of gas may be asymptomatic and must be interpreted cautiously alongside all other available data including clinical examination, clinical laboratory testing, gas analysis, necropsy examination, and histology results.

Keywords: computed tomography, ultrasound, magnetic resonance imaging, cetacean, decompression sickness, bends, pinniped, gas bubbles

\section{BACKGROUND}

Historically marine mammals have been considered protected from decompression sickness (DCS) due to anatomical, physiological, and behavioral evolutionary adaptations. The most important of these concepts was that lung compression induced pulmonary shunting and altered peripheral blood flow by a combination of dive-induced bradycardia with peripheral vasoconstriction, limited the amount of nitrogen that could cross from the pulmonary alveoli in to blood and from blood in to tissues (Scholander, 1940; Butler and Jones, 1997; Kooyman and Ponganis, 1998). Observations of DCS-like gas and fat embolic lesions in stranded beaked whales (Fernández et al., 2005) and acute and chronic gas embolic lesions in multiple cetacean species (Jepson et al., 2003, 2005) in addition to dysbaric osteonecrosis-like skeletal lesions in sperm whales (Moore and Early, 2004) have ignited a contentious debate with divergent opinions on how marine mammals manage gases during diving (Hooker et al., 2012).

In humans, de novo gas bubble formation is reported under hyperbaric and, with less frequency, hypobaric environmental conditions (Pilmanis et al., 2004; Barak and Katz, 2005). In both scenarios, gas bubbles have the opportunity to form when the environmental pressure of nitrogen reduces more rapidly than its tissue pressure, so that the dissolved tissue nitrogen transitions into its gaseous phase (Pilmanis et al., 2004). DCS is the term used when clinical symptoms of this transition manifest in humans. While bubbles are thought to be the precursor for the development of DCS, particularly if within the arterial circulation (Ljubkovic et al., 2011), the presence of gas bubbles alone does not indicate that DCS has, or will, develop (Brubakk and Eftedal, 2001; Barak and Katz, 2005; Ljubkovic et al., 2011). Symptoms experienced in human DCS cases are very varied and non-specific, and are dependent on where gas bubbles lodge and the degree of tissue damage caused. Tissue damage occurs due to primary or secondary mechanisms. The primary mechanisms are thought to be via vascular occlusion (embolism) resulting in tissue ischemia, and parenchymal damage or joint distension due to extravasated bubbles that expand during ascent, resulting in a space-occupying lesion that causes pain (Kayar et al., 1997; Thom et al., 2011). Secondary mechanisms include activation of the complement cascade or activation of inflammatory pathways (Kayar et al., 1997; Thom et al., 2011). These pathways are an area of great interest in human DCS studies and are triggered via endothelial damage and the release of microparticles (small vesicles or fragments that are released from stressed or damaged endothelium and circulate in the blood) in response to decompression stress (Barak and Katz, 2005; Brubakk et al., 2005; Ljubkovic et al., 2011; Thom et al., 2011). Multiple risk factors have been modeled to investigate what factors result in the development of DCS in humans subsequent to gas bubble formation, but the process remains poorly understood (Weathersby et al., 1984, 1992; Pilmanis et al., 2004; Barak and Katz, 2005). 


\section{APPLICATION OF DIAGNOSTIC IMAGING FOR ABNORMAL GAS ACCUMULATIONS}

Available diagnostic imaging for use in cetaceans and pinnipeds includes radiography, ultrasound, and magnetic resonance imaging (MRI) and the use of these modalities in marine mammal medicine is not new. Published use of radiography in marine mammal research dates back to the 1960s (Felts and Spurrell, 1966; Sommer et al., 1968) and has been described in the clinical setting (Sweeney, 1990). The use of computer tomography (CT), MRI, and ultrasound in marine mammals has also been described (Brooks et al., 2001; Van Bonn et al., 2001). Radiography describes the use of $\mathrm{x}$-rays to produce an image and digital radiography, by this definition, includes direct radiography (DR), computed radiography (CR) and CT. Each of these diagnostic imaging modalities produces an image via different methods, and a basic understanding of the physics including limitations and potential pitfalls, is needed so that the modalities are applied appropriately. Size and weight limitations exist and the use of diagnostic imaging in larger cetaceans or even large pinnipeds may be limited or even impossible. When considering imaging dead animals as part of a post mortem investigation, only fresh-dead, intact cadavers should be considered as appropriate for evaluation for gas. If disarticulation is needed to accommodate imaging, or if decomposition has begun, the presence of any gas observed on images becomes questionable. However other information may still be acquired from imaging such cases that could help determine the cause of death.

\section{DIRECT RADIOGRAPHY (DR) AND COMPUTED RADIOGRAPHY (CR)}

DR and CR have increased the sensitivity for detection of small volume gas accumulations compared to conventional screen-film radiography (Marolf et al., 2008). Despite this, superimposition of structures, the need for relatively large gas accumulations for detection, and practical aspects (maneuvering large marine mammals to position radiographic plates, production of not insignificant amounts of scatter radiation in larger animals, and difficulties in achieving adequate $\mathrm{kVp}$ for penetration) often limit the use of DR and CR. Appropriate applications do exist in smaller sized pinnipeds and cetaceans, and successful identification of abnormal gas has been published (Van Bonn et al., 2011). Because of the limitations, DR and CR will not be discussed further.

\section{ULTRASOUND}

Diagnostic ultrasound utilizes sound in the realm of $2-20 \mathrm{MHz}$. The sound beam produced by the piezoelectric transducer penetrates the patient and is reflected at interfaces created by different structures or substances. The proportion of the sound beam reflected is relative to the differences in acoustic impedance of the substances at interfaces (Zagzebski, 1996; Drost, 2007). The greatest difference in acoustic impedance is created at gas-fluid and gas-tissue interfaces and results in near perfect reflection of the sound beam. This causes specific artifacts to be created and displayed on the gray-scale image (Zagzebski, 1996). In the case of large accumulations of gas, reverberation artifacts with equally spaced repetitive bright lines are observed, Figure 1. Furthermore, gas bubbles present as a foam produce a specific subtype of reverberation artifact referred to as ring-down artifact, Figure 2, where

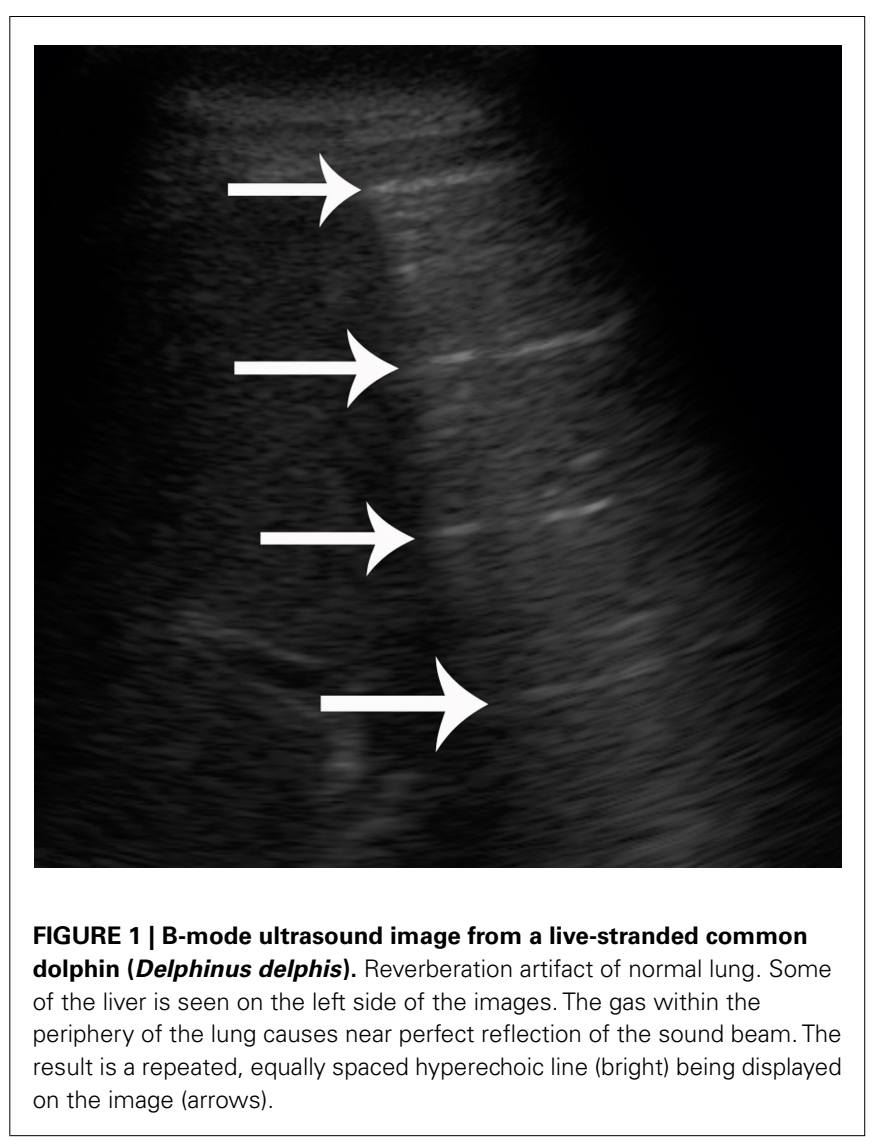

sound bounces between the small gas bubbles before finally being reflected back to the transducer. This artifact is identified by a lack of tapering toward the bottom of the image. Larger gas bubbles present as individuals or in small clusters are often observed as bright foci on the image and may or may not produce reverberation artifact (Kirberger, 1995; Feldman et al., 2009). B-mode ultrasound can be used to detect both stationary and moving gas bubbles.

Doppler ultrasound measures the frequency shift of reflectors as they pass through the sound beam by application of the Nyquist equation (Zagzebski, 1996; Drost, 2007). The frequency shift is within the audible range and can be assessed qualitatively as sound or observed quantitatively as a tracing on the computer display. Most modern ultrasound machines can provide both data simultaneously to improve sensitivity. Pulse-wave Doppler is typically used to evaluate intravascular blood flow. When intravascular gas bubbles traveling with the blood pass through the sound beam, the high difference in acoustic impedance between the gas bubble and the surrounding blood results in a high frequency peak on the pulse-wave tracing and an audible "chirp" that is distinguishable from the background sound of the moving red blood cells (Gillis et al., 1968; Kirberger, 1995; Feldman et al., 2009). Pulsewave Doppler is only applicable in the case of abnormal gas bubble formation, in the detection of circulating gas bubbles.

B-mode and pulse-wave Doppler ultrasound have been used successfully in the research setting to evaluate for the presence of bubbles in human divers (Gillis et al., 1968; Ljubkovic et al., 


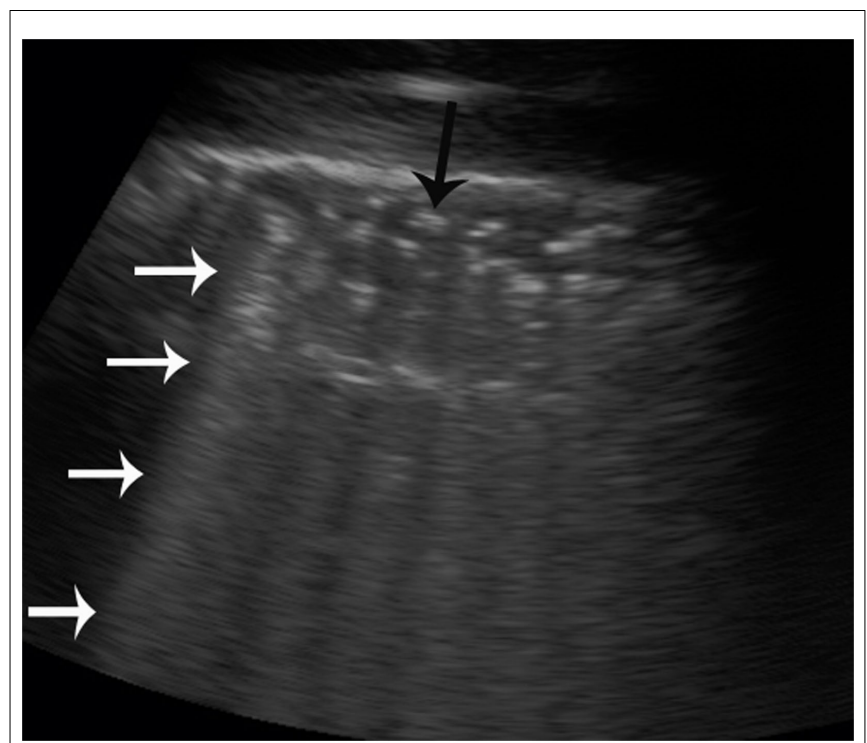

FIGURE 2 | B-mode ultrasound image of a live-stranded common dolphin (Delphinus delphis) kidney. Multiple hyperechoic (bright) foci are observed at the top of the image (black arrow). Ring-down artifact is seen (white arrows) as repeating hyperechoic (bright) lines that do not taper or diminish toward the bottom of the image, confirming that gas is present.

2011), in the clinical setting for cases of suspected air embolism of any etiology including DCS, and in contrast echocardiography studies where stabilized microbubbles are injected intravenously (Bonagura and Pipers, 1983; Stewart, 2003; Miller et al., 2008) B-mode, pulse-wave Doppler and combined B-mode and pulsewave Doppler methodologies are comparable for intravascular gas bubble detection and quantification (Brubakk and Eftedal, 2001).

Highly portable ultrasound machines that can run from battery power and be easily transported are fairly widely available. These units are typically the size of a laptop computer, but hand-held devices are also being developed. This makes ultrasound highly desirable particularly in the case of live-stranded cetaceans where delays in relocation and release may be detrimental and units can be taken directly to the side of the animal. Ultrasound also has a place in the recently dead animal pre-necropsy when advanced cross-sectional imaging modalities (CT or MRI) are not available to help determine if abnormal gas is present prior to dissection.

Several limitations exist when using ultrasound. Depth of penetration is limited and is dependent on the transducer being used. For example, a transducer operating at $4 \mathrm{MHz}$ typically has a maximal depth of penetration of approximately $25 \mathrm{~cm}$, allowing evaluation of the kidneys in pinnipeds, bottlenose dolphins, and beluga and pilot whales, while for a $12-\mathrm{MHz}$ transducer the maximal depth may be as low as $6 \mathrm{~cm}$, allowing evaluation of the eye. Even ignoring the depth limitations, it is not possible for the entire animal to be thoroughly evaluated via ultrasound for gas as regions that normally contain gas, such as the gastrointestinal tract and lungs, prevent evaluation of structures that lie deep to them. When evaluating for abnormal gas accumulations, only regions that do not normally contain gas and lie fairly superficially can be assessed. The most easily accessible and identifiable organs that lie peripherally are the blubber, superficial musculature, eyes, liver, and kidneys (Dennison et al., 2012).

\section{COMPUTED TOMOGRAPHY}

Like DR and CR, CT utilizes x-rays to produce an image with tomography referring to the fact that images are acquired as slices or sections. CT is superior to conventional screen-film CR and DR as superimposition of structures is almost eliminated, depending on operator-selected parameters. CT has greater sensitivity for small differences in attenuation that allows distinction of structures that are indistinguishable on $\mathrm{DR}, \mathrm{CR}$, or conventional screen-film radiography (Gore et al., 2000; Bushberg et al., 2002). Different generations of CT scanner exist that utilize varying technologies but the typical scanners currently in use have a CT tube and detector(s) that circle the patient whom is positioned within the gantry. Beams of $x$-rays pass through the patient through $360^{\circ}$. Different tissues attenuate the $\mathrm{x}$-ray beam to varying degrees and therefore the number of photons ( $\mathrm{x}$-rays) that reach the detector at each scanning point around the patient will be different, determined by the tissues passed through. Once all data for an image have been acquired, reconstruction algorithm selections are applied to produce a recognizable displayed image. Usually reconstruction algorithms for both bone and soft tissue optimization are applied, resulting in the production of two series for the same area. Each pixel in the image has a designated shade of gray that represents an attenuation value. Attenuation is quantitative and is measured in Hounsfield units (HU). Average attenuations for fat are $-100 \mathrm{HU}$, soft tissues are +30 to +50 , and cortical bone are +1000 to $+3500 \mathrm{HU}$ (Bushberg et al., 2002). Essentially gas does not affect the $\mathrm{x}$-ray beam as it passes through, resulting in a very negative $\mathrm{HU}$ value and a pixel coloration that is very dark gray to black, Figure 3.

Computer tomography is able to distinguish between adjacent soft tissue structures better than conventional screen-film radiography, DR, and CR, but contrast between soft tissue structures remains limited using this $\mathrm{x}$-ray based modality. Careful examination and knowledge of CT anatomy is needed to determine the location of gas observed. Determining the exact location of individual accumulations as vascular, parenchymal, or subcapsular, may be difficult, but distinction between normal and abnormal gas accumulations is usually possible. Patterns of distribution can be useful, for example, branching linear accumulations are suggestive of an intravascular location.

The biggest limitations for the use of CT in marine mammals are non-portability that requires the animal be taken to the scanner, and gantry size and table weight limitations that limit the size of animal that can be scanned. Typical CT gantry internal diameter measurements are around $90 \mathrm{~cm}$ but the available scanning field of view is usually less than this. Table weight limitations are around 250-300 kg (Saunders and Ohlerth, 2011) although modified tables designed to support the weight of adult horses and other large animals have been developed. CT tables can usually move approximately $1 \mathrm{~m}$ through the gantry but for longer animals, repositioning mid scan may be necessary if whole body evaluation is desirable. In live cetaceans the dorsal fin can be a limiting size factor and may prevent the caudal thorax/cranial abdomen from passing through the gantry. 


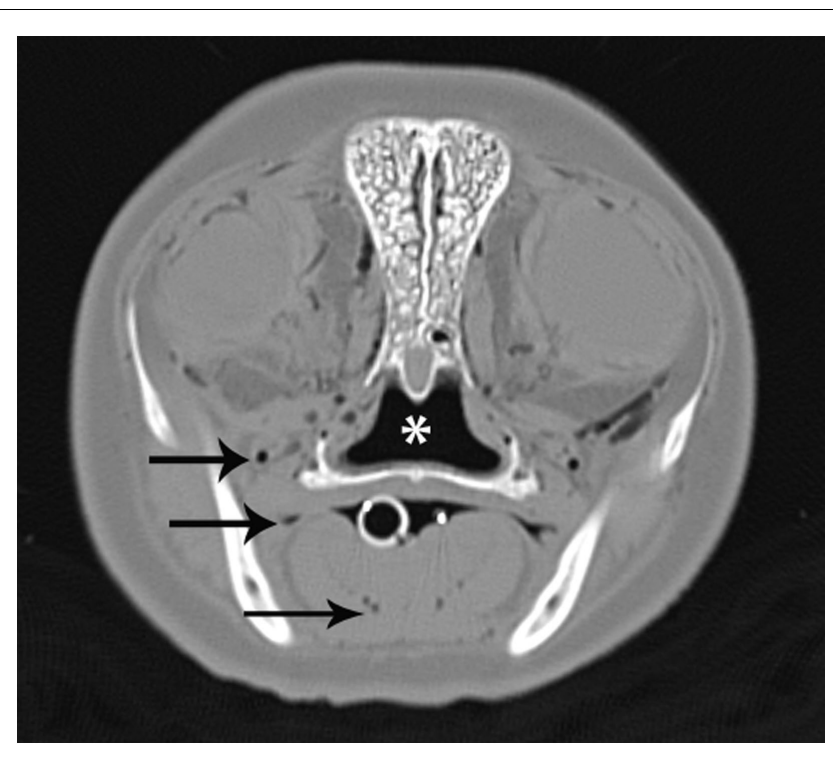

FIGURE 3 | A CT image of the head at the level of the eyes from a bycatch gray seal (Halichoerus grypus). The image was acquired using a bone reconstruction algorithm and displayed on a wide window that results in only gas being displayed as dark gray or black. Normal gas accumulations in the nasopharynx $\left(^{*}\right)$ and oral cavity (white circle) are observed. Abnormal gas accumulations within tissues and vasculature, some demonstrated by black arrows, are also present.

Computer tomography and MRI have both been used to evaluate victims of human diving fatalities prior to autopsy in order to document the extent of gas accumulations and have proven to be invaluable for focusing autopsy efforts (Ozdoba et al., 2005). In clinical medicine, the fast scan time, and high sensitivity for gas makes CT the preferred modality when gas is suspected or its presence needs to be ruled out (Gore et al., 2000; Sebastia et al., 2000; Schindera et al., 2006). For example, CT of the head might take 3-5 min and abdomen 5-10 min depending on the type of scanner. Abnormal gas can be definitively diagnosed from a single scan, unlike in MRI, by measuring the HU of a suspicious area.

\section{MAGNETIC RESONANCE IMAGING}

The greatest advantage of MRI is the contrast this imaging modality affords between and within soft tissue structures, although the use of non-ionizing radiation is also a consideration in live animals. MRI detects signals or energy released from hydrogen within water molecules (Bushberg et al., 2002; Tidwell, 2007). Retrospective manipulation of raw data into alternative sequences is not possible with MRI and therefore appropriate sequence selection prior to scanning is vitally important. Furthermore, multiple sequences in multiple planes are needed for a complete evaluation. Different MRI sequences are used to exploit different properties of the water contained within tissues, which means that the same tissue will often have different signal intensities or shade of gray applied, among different sequences (Tidwell, 2007). Gas contains very few protons and produces a signal void among all MRI sequences represented by black regions on the final images (Tidwell, 2007). Magnetic field inhomogeneities exist at air-tissue interfaces on some sequences, particularly gradient echo sequences. This results in the production of a susceptibility artifact (Rabushka and Kuhlman, 1994; Bushberg et al., 2002) and theoretically allows small gas accumulations to be identified when susceptibility-sensitive sequences are selected.

Care in interpretation of susceptibility artifact is needed, as its presence is not specific to gas. Any substance that results in local inhomogeneities in the magnetic field such as gas, metal, or deoxygenated blood can produce susceptibility artifact on gradient echo sequences (Bushberg et al., 2002; Zhuo and Gullapalli, 2006). This is particularly important to consider in cadaver imaging where deoxygenated blood is to be expected. Blood and gas can be distinguished by comparing different sequences.

Similar to CT, MRI gantry size limitations exist, and units are static requiring that the animal be transported to the scanner. Higher Tesla magnets (1-3 T) that produce high quality images are closed gantry magnets and diameter is typically similar to CT gantries at up to $90 \mathrm{~cm}$. Large bore magnets are becoming available and are particularly geared toward facilitating equine imaging. Open magnets are inherently low Tesla magnets compared to closed magnet configuration (Bushberg et al., 2002) and that affects scan time (making it longer), image quality (lowered), and sequences available (affecting full characterization of pathology observed). However, open magnets may permit imaging in, for example, live cetaceans where the dorsal fin would otherwise be size-prohibitive.

In order to maximize information gained from an MRI study, different sequences in different planes are needed. For MRI a separate scan is required for each sequence and each plane of acquisition, unlike CT. Thus when using MRI each region may require a scan time of $30 \mathrm{~min}$ depending on the sequences acquired and the desired amount of coverage. This may increase in live animals if contrast medium is administered. Dedicated animal MRI scanners are available in academic institutions and commercially, and some MRI availability through human imaging centers may be negotiable. However the availability of MRI is generally less than $\mathrm{CT}$ in veterinary medicine at this time.

Normal accumulations of gas may obscure adjacent abnormal gas on MRI due to susceptibility artifact ad resolution limitations, Figure 4. Cases have been published, however, where MRI has been successful at identifying intestinal wall gas (pneumatosis intestinalis) in intestines that contain normal luminal gas (Rabushka and Kuhlman, 1994). Cerebellar gas has been successfully diagnosed using MRI in a California sea lion (Van Bonn et al., 2011).

\section{APPLICATION OF DIAGNOSTIC IMAGING IN DETERMINING THE MECHANISM OF SUPERSATURATION}

The suggestion that supersaturation is occurring in marine mammals under normal circumstances is contrary to historical understanding of marine mammal physiology. Early studies had concluded that lung compression and alveolar collapse, and associated pulmonary shunting terminating gas-exchange, occurred between 25 and $30 \mathrm{~m}$ in pinnipeds (Falke et al., 1985) and at around $70 \mathrm{~m}$ in cetaceans (Ridgway and Howard, 1979). However if supersaturation is occurring it suggests that our understanding of how marine mammals manage gases during diving is poor. Previously it has been shown that compression of the respiratory system and 


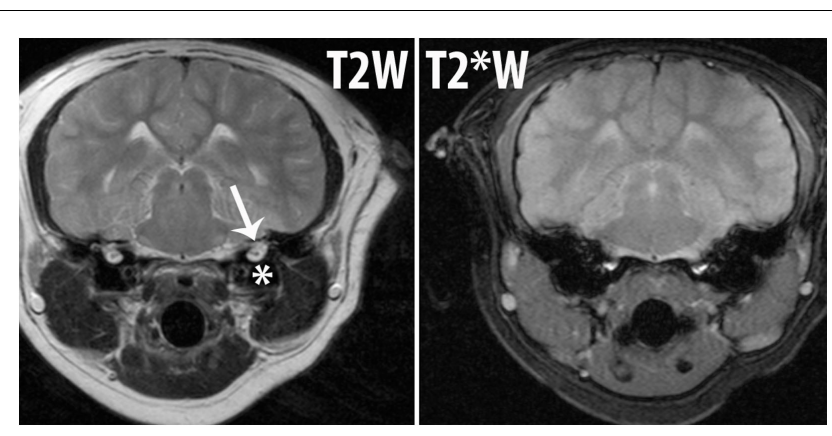

FIGURE 4 | Magnetic resonance imaging images from a California sea lion (Zalophus californianus) to demonstrate the appearance of gas and the associated susceptibility artifact. Both images are at the same level on the same patient. T2W is a regular MRI sequence and $T 2{ }^{*} \mathrm{~W}$ is sensitive to susceptibility artifact. The air within the bulla $\left(^{*}\right)$ on the T2W image can be seen and the inner ear structures (arrow) are also present. The same image is shown after T2* $\mathrm{W}$ acquisition and the amount of gas within and overall size of the bulla looks larger and the "blooming" effect of the susceptibility artifact due to an air-tissue interface results in loss of conspicuity of the inner ear structures. If abnormal gas were present in the inner ear, it would not be identifiable from this image.

pulmonary shunt that develops with depth (Kooyman and Sinnett, 1982) results in tissue and blood gas tensions that are higher than expected. A recent study using a custom-built hyperbaric CT chamber large enough to hold a small marine mammal, was performed to assess the effect of pressure on the respiratory system (Moore et al., 2011). Results estimated that the depth for complete alveolar collapse, or pulmonary shunting, in the gray seal to be $58 \mathrm{~m}$ when the dive began with a diving lung volume of $50 \%$ of total lung capacity. In the harbor porpoise, when the lungs were inflated to total lung capacity prior to the start of the dive, the collapse depth was estimated at $133 \mathrm{~m}$. While the use of cadavers does have limitations, the results concurred with empirical (Kooyman and Sinnett, 1982; Fahlman et al., 2011) and theoretical (Fahlman et al., 2009) data, suggesting that the depth at which pulmonary shunting occurs and gas-exchange terminates is affected by lung volume and is likely more varied and often deeper than previously accepted. Importantly, this suggests that in animals diving with full or near full lung volume, a portion of the dive may occur where the pressure allows a significant amount of nitrogen to be taken up before gas-exchange terminates. This scenario would accommodate development of a supersaturated state and could explain the underlying mechanism permitting de novo gas bubble formation.

\section{APPLICATION OF DIAGNOSTIC IMAGING TO IDENTIFY IN VIVO GAS BUBBLES IN MARINE MAMMALS}

Several studies have been performed in marine mammals to determine if gas bubbles exist using a variety of diagnostic imaging modalities (Houser et al., 2001; Moore et al., 2009; Dennison et al., 2012). Pulse-wave Doppler, B-mode ultrasound, and CT have all been utilized and results among groups of animals, particularly free-ranging vs. captive-maintained animals, have been confounding.

A study of bycaught phocids and cetaceans that died at depth and were hauled to the surface in gill nets demonstrated generalized, diffuse, and severe gas bubble formation within soft tissues, lipid-rich tissues, vasculature, and synovial, cerebrospinal, ocular and lymphatic fluids using CT, necropsy, and histological examinations (Moore et al., 2009). A pathological cause for the vast gas accumulations observed was not identified during necropsy or histopathology. Post mortem off-gassing of supersaturated tissues and fluids during ascent to sea level was hypothesized as the most likely etiology of the gas bubble accumulations. It is certainly possible that some of the bubbles observed were a result of putrefaction, but the extent of bubbling substantially exceeded that of stranded animals of similar time post mortem when examined, and histology did not demonstrate significant autolysis.

Following on from the bycatch study results, stranded freeranging cetaceans were identified as a study cohort that, because of the animals' disrupted dive pattern with loss of the opportunity for recompression, would provide an optimized environment for in vivo, de novo gas bubble formation in live animals, if gas bubble formation due to supersaturation occurs (Dennison et al., 2012). Evaluation of the kidneys, selected as easily accessible and identifiable organs using B-mode diagnostic ultrasound that should not normally contain gas, demonstrated bilateral subcapsular and intrarenal gas accumulations unanimously among the stranded cetaceans evaluated. The exact location of the gas bubbles (subcapsular vs. parenchymal vs. vascular) could not be determined using ultrasound in those cases. CT and necropsy examinations of animals that died or were euthanized within that cohort not only confirmed the renal gas as subcapsular and intravascular, but also identified abnormal gas accumulations in non-renal intravascular locations and within the subdermal sheath. It is unknown if the multifocal, multicompartmental accumulations in those cadavers represented underestimation of gas bubble load via renal ultrasound, that the greater bubble load was related to the ultimate demise of those individuals, or that gas production in those cases progressed in the time between ultrasound, CT, and necropsy. Regardless, the data demonstrated that gas bubble formation does occur in cetaceans under certain circumstances. Several stranded cetaceans with renal gas identified by ultrasound in that study were released uneventfully and some were monitored in the short term using satellite telemetry. Despite the presence of renal gas while stranded, the successful outcome of those free-ranging dolphins suggests that the gas bubble accumulations observed were asymptomatic.

Comparison groups were utilized by Dennison et al. (2012) in an attempt to determine if gas bubble formation only occurred in animals with the ability to undertake significant dives: a fact that would add credence to the hypothesis that gas bubble formation occurs in supersaturated individuals. Shallow water dwelling, free-ranging cetaceans, captive-maintained cetaceans, and stranded pinnipeds also underwent renal ultrasound, but no evidence of renal gas was identified in these groups. These negative data concurred with a separate study performed in a single captive-maintained dolphin that underwent forced repetitive dives over a short period of time (Houser et al., 2010). In that study, pulse-wave Doppler did not demonstrate gas within intrahepatic portal veins or brachiocephalic veins. The differing "life-styles" of the captive-maintained and shallow-dwelling freeranging cetaceans compared to the free-ranging, live-stranded 
dolphins may provide a valid explanation for the negative gas results: insufficient opportunity for chronic supersaturation of tissues to develop in shallow-dwelling cetaceans.

Barotrauma occurs when pressure alterations result in damage to the tissues surrounding air-filled cavities. This is because the air is compressible while the surrounding tissue is not. If the damage permits entrance of compressed air into the vascular system while still at depth, air can travel to an abnormal location, then expand to form a "space-occupying" lesion resulting in tissue damage as decompression occurs. Imaging such animals prior to necropsy provides invaluable information regarding distribution and degree of gas bubble formation, much of which may not be directly observed during necropsy without disruption or dissection of tissues.

Barotrauma is a valid differential diagnosis when gas bubbles are identified in an individual particularly if there is evidence of concurrent damage to a gas filled cavity or lung tissue. Cases of diagnosed marine mammal barotrauma evaluated with diagnostic imaging have been published. Pneumocerebellum developed in a neurologically abnormal, stranded, free-ranging California sea lion with additional evidence of rib fracture and pneumothorax (Van Bonn et al., 2011). Here cerebellar gas accumulations were speculated to have developed when circulating gas bubbles introduced into the vascular system from the damaged pulmonary tract traveled to and became entrapped within the cerebellar parenchyma while still at depth. The bubbles then expanded during resurfacing. Gas was recognized within the caudal fossa using standard radiography but further characterization (intraparenchymal, subarachnoid, subdural, epidural) was not possible. The large intraparenchymal cerebellar gas cavitations were clearly evident on all MRI sequences as signal voids with susceptibility artifact on susceptibility - sensitive sequences.

Abnormal gas accumulations are non-specific for the type of gas present and as such, imaging alone cannot distinguish decomposition from disarticulation/dissection from de novo formation. It is possible that as more cases are fully evaluated, gas bubble distributions associated with the different etiologies may be distinguishable. Even then, confirmation of the likely etiology based on the type of gas present and its likely significance will need a combined approach including imaging, gas analysis, and histology. Of course, this will not be possible in the live animal, but barotrauma victims would not be expected to present as otherwise healthy individuals with normal behavior unlike the dolphins with presumed asymptomatic renal gas described (Dennison et al., 2012). Evidence of pneumothorax, pneumoperitoneum or damage to normally gas-filled structures may explain abnormal gas accumulations in some cases but distinguishing between trauma or barotrauma is difficult from imaging alone. Crossover may exist and in supersaturated animals that sustain barotrauma or other trauma to gas-filled structures, abnormal gas accumulations due to concurrent processes is quite feasible.

\section{THE SIGNIFICANCE OF ABNORMAL GAS ACCUMULATIONS DEMONSTRATED VIA DIAGNOSTIC IMAGING IN MARINE MAMMALS}

The formation of gas bubbles in marine mammals may in many cases be asymptomatic and as such the presence of gas alone must be interpreted with caution. The live-stranded, free-ranging cetacean renal gas accumulations observed with B-mode ultrasound were considered entrapped or "safe" with presumption that they were interstitial in location, or more specifically were not arterial in location which is maximally detrimental with respect to the likelihood of the development of DCS and resultant damage due to vascular occlusion (Brubakk and Eftedal, 2001; Barak and Katz, 2005; Dennison et al., 2012). However vascular gas bubbles have been observed on CT images and during necropsy examinations in dead stranded animals that were identified as positive for renal gas while still alive (Dennison et al., 2012), suggesting that some gas may be circulating. Vascular studies in this group of animals to confirm this have not yet been performed.

Intravascular circulating gas bubbles can theoretically lodge anywhere in the body, limited only by their size. While there is the temptation to say that very small bubbles are therefore innocuous, in the biological setting it is possible that small bubbles could pass through protective capillary beds, particularly if the bubbles are partially compressed, only to occlude larger vessels if they coalesce to form larger bubbles or expand during final ascent. Bubbles entering the arterial side of circulation in humans have been associated with development of DCS (Ljubkovic et al., 2011).

Circulating gas bubbles of any size that come into contact with endothelium during their travels can activate inflammatory or complement pathways via release of microparticles or directly damage endothelial cells as they pass (Barak and Katz, 2005; Thom et al., 2011). Studies have shown that endothelial damage is related to bubble load in humans (Brubakk and Eftedal, 2001). The probability of contact between bubbles and endothelium is theoretically greater in smaller gage blood vessels such as capillaries than in larger gage vessels where the volume of blood and laminar blood flow may help distance bubbles from the endothelial cells. Typically in humans, the lungs act as a large filter, removing many bubbles from the circulation but when high bubble load is present, arterial gas bubbles are seen with some frequency even in the absence of congenital heart defects such as patent foramen ovale (Ljubkovic et al., 2011). Given the identification of renal gas bubbles in marine mammal, the kidneys, or its adjacent vasculature may be functioning as a filter or possibly as a method of detection. It is likely that other anatomical, physiological, or behavioral mechanisms also play a role in protection against gas bubble formation or the prevention of deleterious effects.

Given the temporal and spatial association between beaked whale strandings and anthropogenic noise (Jepson et al., 2003; Fernández et al., 2005; D'Amico et al., 2009), the potential effects of anthropogenic noise on marine mammals deserve special consideration. The concern for auditory effects of anthropogenic noise have been voiced following CT and necropsy findings of aural, periaural, and intracranial hemorrhage in beaked whales and have led to imaging recommendations in similar stranding events (Ketten and Montie, 2008). However non-auditory effects also warrant consideration as anthropogenic sound may alter behavior increasing the risk of bubble formation by decompression, or directly initiating or advancing bubble formation in supersaturated tissues through rectified diffusion (Houser et al., 2001). Exposure of bubbles to sound could result in bubble rupture and subsequent tissue damage if sufficient numbers of bubbles are present, 
as has been shown in in vivo studies of isolated rabbit hearts perfused with intravenous stabilized microbubbles (Miller et al., 2008) creating a very high bubble load. Reported tissue damage observed in that study associated with gas bubble rupture included microvascular leakage, petechiae formation, cardiomyocyte death, and premature ventricular arrhythmias. Extrapolating these data, prolonged sound exposure in marine mammals that have formed gas bubbles may, therefore, have serious consequences. Thus, auditory and non-auditory effects of anthropogenic sound should be considered as potential causes of marine mammal strandings particularly when evidence of gas bubbling is present. Furthermore, if utilizing B-mode ultrasound to evaluate stationary bubbles, when high volumes of gas bubbles are identified, prolonged examination of tissue containing gas as small bubbles using a high mechanical index without movement of the sound beam should be avoided to prevent potentially iatrogenic deleterious effects.

\section{CONCLUSION}

The evidence suggests that supersaturation and gas bubble formation occur in marine mammals. Under normal circumstances anatomical, physiological, and behavioral adaptations must exist to help protect susceptible animals from detrimental effects, and in many cases gas bubble formation is asymptomatic. Diagnostic

\section{REFERENCES}

Barak, M., and Katz, Y. (2005). Microbubbles. Pathological and clinical implications. Chest 128, 2918-2932.

Bonagura, J. D., and Pipers, F. S. (1983). Diagnosis of cardiac lesions by contrast echocardiography. J. Am. Vet. Med. Assoc. 182, 396-402.

Brooks, F., Van Bonn, W., and Jensen, E. (2001). "Ultrasonography," in CRC Handbook of Marine Mammal Medicine, eds L. A. Dierauf and F. M. D. Gulland (Boca Raton, FL: CRC Press), 593-620.

Brubakk, A. O., Duplancic, D., Valic, Z., Palada, I., Obad, A., Bakovic, D., Wisloff, U., and Dujic, Z. (2005). A single air dive reduces arterial endothelial function in man. J. Physiol. (Lond.) 566, 901-906.

Brubakk, A. O., and Eftedal, O. (2001). Comparison of the three different ultrasonic methods for quatification of intravascular gas bubbles. Undersea Hyperb. Med. 28, 131-136.

Bushberg, J. R. T., Seibert, J. A., Leidholdt, E. M., and Bonne, J. M. (2002). The Essential Physics of Medical Imaging. Philadelphia: Lippincott Williams and Wilkins.

Butler, P. J., and Jones, D. R. (1997). Physiology of diving birds and mammals. Physiol. Rev. 77, 837-899.

D’Amico, A. D., Gisiner, R., Ketten, D. R., Hammock, J. A., Johnson, C., Tyack, P., and Mead, J. (2009). Beaked whale strandings and naval exercises. Aquat. Mamm. 35, 452-472.
Dennison, S., Moore, M. J., Fahlman, A., Moore, K., Sharp, S., Harry, C. T., Hoppe, J., Niemeyer, M., Lentell, B., and Wells, R. S. (2012). Bubbles in live-stranded dolphins. Proc. R. Soc. Lond. B Biol. Sci. 79, 1396-1404.

Drost, W. T. (2007). "Basic ultrasound physics," in Textbook of Veterinary Diagnostic Imaging, ed. D. 38-49.

Fahlman, A., Hooker, S. K., Olszowka, A., Bostrom, B. L., and Jones, D. R. (2009). Estimating the effect of lung collapse and pulmonary shunt on gas exchange during breath-hold diving: the Scholander and Kooyman legacy. Respir. Physiol. Neurobiol. 165, 28-39.

Fahlman, A., Loring, S. H., Ferrigno, M., Moore, C., Early, G., Niemeyer, M., Lentell, B., Wenzel, F., Joy, R., and Moore, M. J. (2011). Static inflation and deflation pressurevolume curves from excised lungs of marine mammals. J. Exp. Biol. 214, 3822-3828.

Falke, K. J., Hill, R. D., Qvist, J., Schneider, R. C., Guppy, M., Liggins, G. C., Hochachka, P. W., Elliot, R. E., and Zapol, W. M. (1985). Seal lung collapse during free diving: evidence from arterial nitrogen tensions. Science 229, 556-557.

Feldman, M. K., Katyal, S., and Blackwood, M. S. (2009). US artifacts. Radiographics 29, 1179-1189.

Felts, J. L., and Spurrell, F. A. (1966). Some structural and developmental Thrall (St. Louis: Saunders Elsevier),

imaging can be useful to determine if gas bubbles are present. Ultrasound is a highly portable and easily accessible imaging modality that can be applied to live-stranded animals on the beach, but the use of ultrasound in the live animal may underestimate abnormal gas distribution and bubble load. CT or MRI studies of dead marine mammals pre-necropsy where possible will allow better documentation of the distribution and bubble load present than can be achieved with necropsy observations alone, but the application of these modalities in the live-stranded animal is likely to remain very limited. Comparisons between distributions of gas accumulations identified via different imaging modalities from cases determined as most likely due to or know to be barotrauma, decomposition, or bycatch from unknown causes presumed to be de novo gas formation may help separate out etiologies based on imaging characteristics. This would require correlation with histopathology and gas analyses. Future studies should develop deployable field imaging techniques to determine if spontaneous gas bubble formation occurs in marine mammals that are not entering a disturbed or stranded state. In addition, further imaging studies in live-stranded cetaceans are needed to determine if gas bubbles exist both in tissues and the vasculature incidentally, what bubble load or distribution may have detrimental effects and under what circumstances such detrimental accumulations occur.

characteristics of cetacean (Odontocete) radii. A study of adaptive osteogenesis. Am. J. Anat. 118 103-133.

Fernández, A., Edwards, J. F., Rodriguez, F., Espinosa De Los Monteros, A., Herraez, P., Castro, P., Jaber, J. R., Martin, V., and Arbelo, M. (2005). 'Gas and fat embolic syndrome' involving a mass stranding of beaked whales (family Ziphiidae) exposed to anthropogenic sonar signals. Vet. Pathol. 42, 446-457.

Gillis, M. F., Peterson, P. L., and Karagianes, M. T. (1968). In vivo detection of circulating gas emboli associated with decompression sickness using the Doppler flowmeter. Nature 217, 965-967.

Gore, R. M., Miller, F. H., Pereles, F. S, Yaghmai, V., and Berlin, J. W. (2000). Helical CT in the evaluation of the acute abdomen. Am. J. Radiol. 174, 901-913.

Hooker, S. K., Fahlman, A., Moore, M. J., Aguilar De Soto, N., Bernaldo De Quiros, Y., Brubakk, A. O., Costa, D. P., Costidis, A. M., Dennison, S. Falke, K. J., Fernandez, A., Ferrigno, M., Fitz-Clarke, J. R., Garner, M. M. Houser, D. S., Jepson, P. D., Ketten, D. R., Kvadsheim, P. H., Madsen, P. T., Pollock, N. W., Rotstein, D. S., Rowles, T. K., Simmons, S. E., Van Bonn, W., Weathersby, P. K., Weise, M. J., Williams, T. M., and Tyack, P. L. (2012). Deadly diving? Physiological and behavioural management of decompression stress in diving mammals. Proc. R. Soc. Lond. B Biol. Sci. 279, 1041-1050.

Houser, D. S., Dankiewicz-Talmadge, L. A., Stockard, T. K., and Ponganis, P. J. (2010). Investigation of the potential for vascular bubble formation in a repetitively diving dolphin. J. Exp. Biol. 213, 52-62.

Houser, D. S., Howard, R., and Ridgway, S. (2001). Can divinginduced tissue nitrogen supersaturation increase the chance of acoustically driven bubble growth in marine mammals? J. Theor. Biol.213, 183-195.

Jepson, P. D., Arbelo, M., Deaville, R., Patterson, I. A. P., Castro, P., Baker, J. R., Degollada, E., Ross, H. M., Herraez, P., Pocknell, A. M., Rodriguez, F., Howie, F. E., Espinosa, A., Reid, R. J., Jaber, J. R., Martin, V., Cunningham, A. A., and Fernandez, A. (2003). Gas-bubble lesions in stranded cetaceans. Nature 425 , 575-576.

Jepson, P. D., Deaville, R., Patterson, I. A. P., Pocknell, A. M., Ross, H. M., Baker, J. R., Howie, F. E., Reid, R. J., Colloff, A., and Cunningham, A. A. (2005). Acute and chronic gas bubble lesions in cetaceans stranded in the United Kingdom. Vet. Pathol. 42 , 291-305.

Kayar, S. R., Aukhert, E. O., Axley, M. J., Homer, L. D., and Harabin, A. L. (1997). Lower decompression sickness risk in rats by intravenous injection of foreign protein. Undersea Hyperb. Med. 24, 329-335. 
Ketten, D. R., and Montie, E. W. (2008). Imaging Procedures for Stranded Marine Mammals. Woods Hole: Woods Hole Oceanographic Institution Technical Publications.

Kirberger, R. M. (1995). Imaging artifacts in diagnostic ultrasound - a review. Vet. Radiol. Ultrasound 36, 297-306.

Kooyman, G. L., and Ponganis, P. J. (1998). The physiological basis of diving to depth: birds and mammals. Annu. Rev. Physiol. 60, 19-32.

Kooyman, G. L., and Sinnett, E. E. (1982). Pulmonary shunts in Harbor sels and sea lions during simulated dives to depth. Physiol. Zool. 55, 105-111.

Ljubkovic, M., Dujic, Z., Mollerlokken, A., Bakovic, D., Obad, A., Breskovic, T., and Brubakk, A. O. (2011). Venous and arterial bubbles at rest after no decompression air dives. Med. Sci. Sports Exerc. 43, 990-995.

Marolf, A., Blaik, M., Ackerman, N., Watson, E., Gibson, N., and Thompson, M. (2008). Comparison of computed radiography and conventional radiography in detection of small volume pneumoperitoneum. Vet. Radiol. Ultrasound 49, 227-232.

Miller, D. L., Averkiou, M. A., Brayman, A. A., Everbach, E. C., Holland, C. K., Wible, J. H., and Wu, J. (2008). Bioeffects coniderations for diagnostic ultrasound contrast agents. J. Ultrasound Med. 27, 611-632.

Moore, M. J., Bogomolni, A. L., Dennison, S. E., Early, G., Garner, M. M., Hayward, B. A., Lentell, B. J., and Rotstein, D. S. (2009). Gas bubbles in seals, dolphins, and porpoises entangled and drowned at depth in gillnets. Vet. Pathol. 46, 536-547.

Moore, M. J., and Early, G. A. (2004). Cumulative sperm whale bone damage and the bends. Science 306, 2215.
Moore, M. J., Hammar, T., Arruda, J., Cramer, S., Dennison, S., Montie, E., and Fahlman, A. (2011). Hyperbaric computed tomographic measurement of lung compression in seals and dolphins. J. Exp. Biol. 214, 2390-2397.

Ozdoba, C., Weis, J., Plattner, T., Dirnhofer, R., and Yen, K. (2005). Fatal scuba diving incident with massive gas embolism in cerebral and spinal arteries. Neuroradiology 47, 411-416.

Pilmanis, A. A., Petropoulos, L. F., Kannan, N., and Webb, J. T. (2004). Decompression sickness risk model: development and validation by 150 prospective hypobaric exposures. Aviat. Space Environ. Med. 75, 749-759.

Rabushka, L. S., and Kuhlman, J. E. (1994). Pneumatosis intestinalis: appearance on MR examination. Clin. Imaging 18, 258-361.

Ridgway, S. H., and Howard, R. (1979). Dolphin lung collapse and intramuscular circulation during free diving: evidence from nitrogen washout. Science 206, 1182-1183.

Saunders, J., and Ohlerth, S. (2011). "CT physics and instrumentation - mechanical design," in Veterinary Computed Tomography, eds T. Schwarz and J. Saunders (Chichester: Wiley-Blackwell), 1-8.

Schindera, S. T., Triller, J., Vock, P., and Hoppe, H. (2006). Detection of hepatic portal venous gas: its clinical impact and outcome. Emerg. Radiol. 12, 164-170.

Scholander, P. F. (1940). Experimental investigations on the respiratory function in diving mammals and birds. Hvalrådets Skrifter 22, 1-131.

Sebastia, C., Quiroga, S., Espin, E., Boye, R., Alvarez-Castells, A., and
Armengol, A. (2000). Portomesnetric vein gas: pathologic mechanisms, CT findings and prognosis. Radiographics 20, 1213-1224.

Sommer, L. S., Mcfarland, W. I., Galliano, R. E., Nagel, E. L., and Morgane, J. P. (1968). Hemodynamics and coronary angiographic studies in the bottlenose dolphin (Tursiops truncatus). Am. J. Physiol. 215, 1498-1505.

Stewart, M. J. (2003). Contrast Echocardiography. Heart 89, 342-348.

Sweeney, J. C. (1990). "California sea lion radiology," in Handbook of Marine Mammal Medicine: Health, Disease and Rehabilitation, ed. L. A. Dieuraf (Boca Raton, FL: CRC Press), 203-213.

Thom, S. R., Yang, M., Bhopale, V. M., Huang, S., and Milovanova, T. N. (2011). Microparticles initiate decompression-induced neutrophil activation and subsequent vascular injuries. J. Appl. Physiol. 110, 340-351.

Tidwell, A. (2007). "Principles of computed tomography and magnetic resonance imaging," in Textbook of Veterinary Diagnostic Imaging, ed. D. Thrall (St. Louis, MO: Saunders Elsevier), 56-77.

Van Bonn, W., Jensen, E. D., and Brook, F. (2001). "Radiology, computed tomography and magnetic resonance imaging," in CRC Handbook of Marine Mammal Medicine, eds L. A. Dierauf and F. M. D. Gulland (Boca Raton, FL: CRC Press), 557-591.

Van Bonn, W., Montie, E., Dennison, S., Pussini, N., Cook, P., Greig, D., Barakos, J., Colegrove, K., and Gulland, F. (2011). Evidence of injury caused by gas bubbles in a live marine mammal: barotrauma in a California sea lion Zalophus californianus. Dis. Aquat. Org. 96, 89-96.

Weathersby, P. K., Homer, L. D., and Flynn, E. T. (1984). On the likelihood of decompression sickness. J. Appl. Physiol. 57, 815-825.

Weathersby, P. K., Survanshi, S. S., Homer, L. D., Parker, E., and Thalmann, E. D. (1992). Predicting the time of occurrence of decompression sickness. J. Appl. Physiol. 72, 1541-1548.

Zagzebski, J. A. (1996). Essential of Ultrasound Physics. St. Louis: Mosby.

Zhuo, J., and Gullapalli, R. P. (2006). AAPM/RSNA physics tutorial for residents. Radiographics 26, 275-297.

Conflict of Interest Statement: The authors declare that the research was conducted in the absence of any commercial or financial relationships that could be construed as a potential conflict of interest.

Received: 27 February 2012; accepted: 17 May 2012; published online: 06 June 2012.

Citation: Dennison S, Fahlman $A$ and Moore M (2012) The use of diagnostic imaging for identifying abnormal gas accumulations in cetaceans and pinnipeds. Front. Physio. 3:181. doi: 10.3389/fphys.2012.00181

This article was submitted to Frontiers in Aquatic Physiology, a specialty of Frontiers in Physiology.

Copyright (C) 2012 Dennison, Fahlman and Moore. This is an open-access article distributed under the terms of the Creative Commons Attribution Non Commercial License, which permits noncommercial use, distribution, and reproduction in other forums, provided the original authors and source are credited. 